\title{
Rural Community Members' Perceptions of Harm from Medical Mistakes: A High Plains Research Network (HPRN) Study
}

Rebecca F. Van Vorst, MSPH, Rodrigo Araya-Guerra, BA, Maret Felzien, MA, Douglas Fernald, MA, Nancy Elder, MD, MSPH, Christine Duclos, PhD, MPH, and John M. Westfall, MD, MPH

Objective: The aim of this study was to learn about community members' definitions and types of harm from medical mistakes.

Methods: Mixed methods study using community-based participatory research (CBPR). The High Plains Research Network (HPRN) with its Community Advisory Council (CAC) designed and distributed an anonymous survey through local community newspapers. Survey included open-ended questions on patients' experiences with medical mistakes and resultant harm. Qualitative analysis was performed by CAC and research team members on mistake descriptions and types of reported harm. Patient Safety Taxonomy coding was performed on a subset of surveys that contained actual medical errors.

Results: A total of 286 surveys were returned, with 172 respondents $(60 \%)$ reporting a total of 180 perceived medical mistakes. Quantitative analysis showed that $41 \%$ of perceived mistakes $(n=73)$ involved only unanticipated outcomes. Reported types of harm included emotional, financial, and physical harm. Reports suggest that perceived clinician indifference to unanticipated outcomes may lead to patients' loss of trust and belief that the unexpected outcome was a result of an error.

Discussion: CBPR methodology is an important strategy to design and implement a community-based survey. Community members reported experiencing medical mistakes, most with harmful outcomes. The response they received by the medical community may have influenced their perception of mistake and harm. (J Am Board Fam Med 2007;20:135-143.)

Over the past 5 years, the government, the medical community and health policy experts have focused their attention on improving patient safety. In February 2005, the Agency for Healthcare Research and Quality (AHRQ) released Advances in Patient Safety: From Research to Implementation, the culmi-

Submitted 25 August 2006; revised 19 December 2006; accepted 2 January 2007.

From the Foundation for Health Living, Latham, NY (RFV); the Department of Family Medicine, University of Colorado at Denver and Health Sciences Center, Aurora, CO (RA-G, DF, JMW); HPRN Community Advisory Council, Northeastern Junior College, Sterling, CO (MF); Department of Family Medicine, University of Cincinnati, Cincinnati, $\mathrm{OH}(\mathrm{NE})$; and JSI Research and Training Institute, Denver, CO (CD).

Funding: This study was funded in part by the Agency for Healthcare Research and Quality (Applied Strategies for Improving Patient Safety, Grant U18 HS11878).

Prior presentation: Portions of this work have been presented at the North American Primary Care Research Conference, October 16, 2005, Quebec City, Quebec, Canada.

Conflict of interest: none declared.

Corresponding author: Rebecca F. Van Vorst, MSPH, Foundation for Healthy Living, 30 Century Hill Dr, Latham, NY 12110 (E-mail: vanvorst.rebecca@ foundationforhealthyliving.org). nation of findings from 5 years of governmentfunded research focused on preventing medical errors through the use of successful interventions, advanced technology, and effective policy changes. ${ }^{1}$ The goal of reducing harm caused by medical mistakes now drives the implementation of these changes. $^{2}$ Yet, fewer studies have focused on the patients' views on the safety of health care, the definition of medical errors or the risks associated with medical errors. ${ }^{3-8}$

The use of surveys to elicit patient opinions and perspectives is well described in the literature. ${ }^{9-11}$ Although such surveys have the potential to inform patient safety, most surveys offer patients limited definitions of medical errors ${ }^{12}$ or inquire about specific types of medical events. ${ }^{13}$ Although many studies demonstrate that patients generally report satisfactory experiences with medical care, they also report significant problems with numerous aspects of care. Moreover, most studies were conducted within hospitals or were confined to patients in urban environments. ${ }^{11-17}$ 
The purpose of this study was to use a community-based participatory research (CBPR) approach $^{16,17}$ to develop a community survey and distribution methodology to assess rural community members' experiences with medical mistakes within a practice-based research network (PBRN). CBPR integrates the knowledge and the expertise of the people under study in each phase of the research, ${ }^{18}$ enhancing the research's value and relevance by grounding it in real-life patient experience. ${ }^{19}$ Historically, PBRNs have conducted participatory research with primary care physicians but have not included patients or community members actively in the research process. ${ }^{19}$

\section{Methods}

\section{Study Design}

We used a mixed methods approach to examine how patients define medical mistakes and classify types of harm resultant from these errors. All data were collected by anonymous surveys. We believed that people in their rural communities would be more forthcoming with their experiences of medical mistakes if they could recount their experiences anonymously on a survey than if we had used more traditional qualitative methods such as focus groups. We designed our surveys to include openended questions. Specifically, we asked "Have you or a family member experienced a medical mistake? If yes, please summarize what happened. Do you feel that you or the family member was harmed by this medical mistake? If yes, how were you harmed?" We also collected information about who was involved with the mistake, who had talked to the patient, where the mistake occurred, and the demographics of the patient involved in the mistake.

\section{Use of CBPR for Survey Development}

Using a CBPR approach, the HPRN's research staff and the HPRN's CAC designed the survey to include appropriate language for community members, an easy to follow layout, and devised the dissemination method. The CAC is a group of 10 community members who live and work in the HPRN's rural geographical region in northeastern Colorado and includes farmers, ranchers, educators, and retired administrative workers from various social economic strata, ethnicities, and genders. The CAC's mission is to ground the HPRN's re- search in real patient experience. During survey development, the CAC recommended to change the language of "patient safety event" to "medical mistake." Thus, "medical mistake" will be used throughout this article. The CAC also suggested that we provide a definition of medical mistake. We decided to use a broad definition: "Medical mistakes can be serious (such as a mistake during surgery that may require additional surgery) or not so serious (such as not getting your test results back)." The CAC recommended the distribution method of inserting surveys in 4 rural community newspapers. Their belief was that most people in the rural communities read the local paper. The CAC pilot tested the survey with members of their communities for response burden and for overall understandability of the survey questions. Surveys were designed as a trifolded, prepasted, single sheet of paper. The back two thirds of the survey contained the CAC logo and a business reply, postage paid label and so that once folded, only the logo and the mailing label were visible. All completed surveys were mailed directly to the HPRN research offices.

\section{Data Collection}

We distributed 11,500 surveys via inserts in 4 local newspapers in 4 rural and frontier communities in northeastern Colorado. One week before the survey distribution, an advertisement was placed in each newspaper to alert the community that the survey would be inserted in the following week's newspaper. At least $1 \mathrm{CAC}$ member lived in each of the participating communities. Four CAC members were approached within their community by someone who had seen the survey in the newspaper but wanted another copy. This resulted in the distribution of another 25 surveys.

\section{Data Analysis}

In the analysis of the qualitative data from the open-ended questions, we used a combined template and editing approach. ${ }^{20}$ This facilitates a team approach that interprets the data through themes and issues while acknowledging pre-existing biases and beliefs. Responses were entered into Microsoft Word files, and survey responses were divided into packets of 20 to 30 each and distributed to $10 \mathrm{CAC}$ members and 9 research staff (including physicians, others with doctoral training, and research assistants). Several members of the CAC reviewed more than 1 packet of responses. By the conclusion of the 
analysis, each survey was reviewed by at least 1 CAC member and at least 1 research staff member. For consistency, the principal investigator (PI) and 2 members of the research team reviewed all surveys.

In a joint meeting, both researchers and community members were instructed on how to perform the analysis of the data. Initially, both the 9 members of the research staff and the 10 CAC members individually read through their packets of responses, highlighted particular issues and themes, and identified illustrative quotes. All results of this analysis were sent to the PI for compiling. Then, in a combined coding session, the research staff and the CAC members reviewed the findings, grouped similar issues, and expanded on themes until saturation was reached. Any differences in findings were discussed, and the group negotiated consensus. The PI and a member of the research team then reviewed all the surveys again to ensure that the illustrative quotes related to the themes.

All CAC members reviewed and offered input on this manuscript, and several members presented these data at a national primary care research conference. One member of the CAC participated substantially in the manuscript preparation and is a coauthor (MF).

To analyze the data using a quantitative approach, we used the Applied Strategies for Improving Patient Safety Dimensions of Medical Outcomes (ASIPS DMO) taxonomy. ${ }^{21}$ The ASIPS DMO is a multiaxial taxonomy developed to code medical errors reported by medical personnel in ambulatory practices ${ }^{22}$ and is available for review. ${ }^{23}$ The use of this taxonomy results in a broad understanding of the process of errors and allows for easy grouping of processes across or within specific clinical activity and across various types of processes. ${ }^{24}$ The taxonomy consists of 421 available codes distributed among 10 axes and classifies the setting of each event, the clinical domain (eg, procedures and medications), patient outcome, course and cause(s), discoverer, and roles and contributions of participants involved. ${ }^{21}$ Using this coding schema, 6 members of the research team read each report and by consensus selected 1 or more codes for each dimension that described the nature of the reported event. ${ }^{25}$

Our current study was approved by the Colorado Multiple Institutional Review Board.

\section{Results}

We received 286 surveys with $60 \%(\mathrm{n}=172)$ of the surveys indicating that the respondent or a family member had experienced a medical mistake. Figure 1 depicts the sample and the analysis performed on each subset. Six surveys contained multiple errors yielding a total of 180 perceived mistakes. Table 1 describes the characteristics of these 180 reports. More than half of the surveys involved female patients (59\%), $45 \%$ were sent in by a family member, and $50 \%$ involved patients older than 55 years of age.

Community members were asked to indicate to whom they had talked about the perceived mistake and who was involved by selecting from a list of possible roles. As shown in Table 2, 76\% of respondents talked to a family member or friend, $61 \%$ reported talking to a doctor or clinician, and $26 \%$ talked to a nurse. Seven percent reported that they had not talked to anyone. A doctor or clinician was involved in $80 \%$ of the mistakes, a nurse in $39 \%$, and hospital staff in $33 \%$. Perceived mistakes involved the local hospital and the local physician's office, $44 \%$ and $36 \%$ of the time, respectively.

\section{Type of Reports}

Using the definitions of medical errors from the ASIPS DMO taxonomy, ${ }^{21}$ we classified reported mistakes into 3 major categories: obvious medical mistakes, possible mistakes (events perceived by the reporter to be a mistake but the report did not contain enough information to conclude that a mistake had occurred), and reports that described problems or unanticipated outcomes but contained evidence that this was not truly a mistake. We found that only $30 \%$ of the 180 reported mistakes $(\mathrm{n}=54)$ contained an identifiable medical mistake, $29 \%(\mathrm{n}=53)$ contained possible errors, and $41 \%$ of reports $(\mathrm{n}=73)$ involved only unanticipated outcomes.

\section{Medical Mistake versus Unanticipated Outcome}

We received many types of medical mistakes, including diagnostic delays, missed diagnoses, medication errors, missing information, surgical mistakes and near misses (mistakes that were "caught" before any harm occurred). (Illustrative quotations are presented using the same spelling and punctuation contained in the report.) 


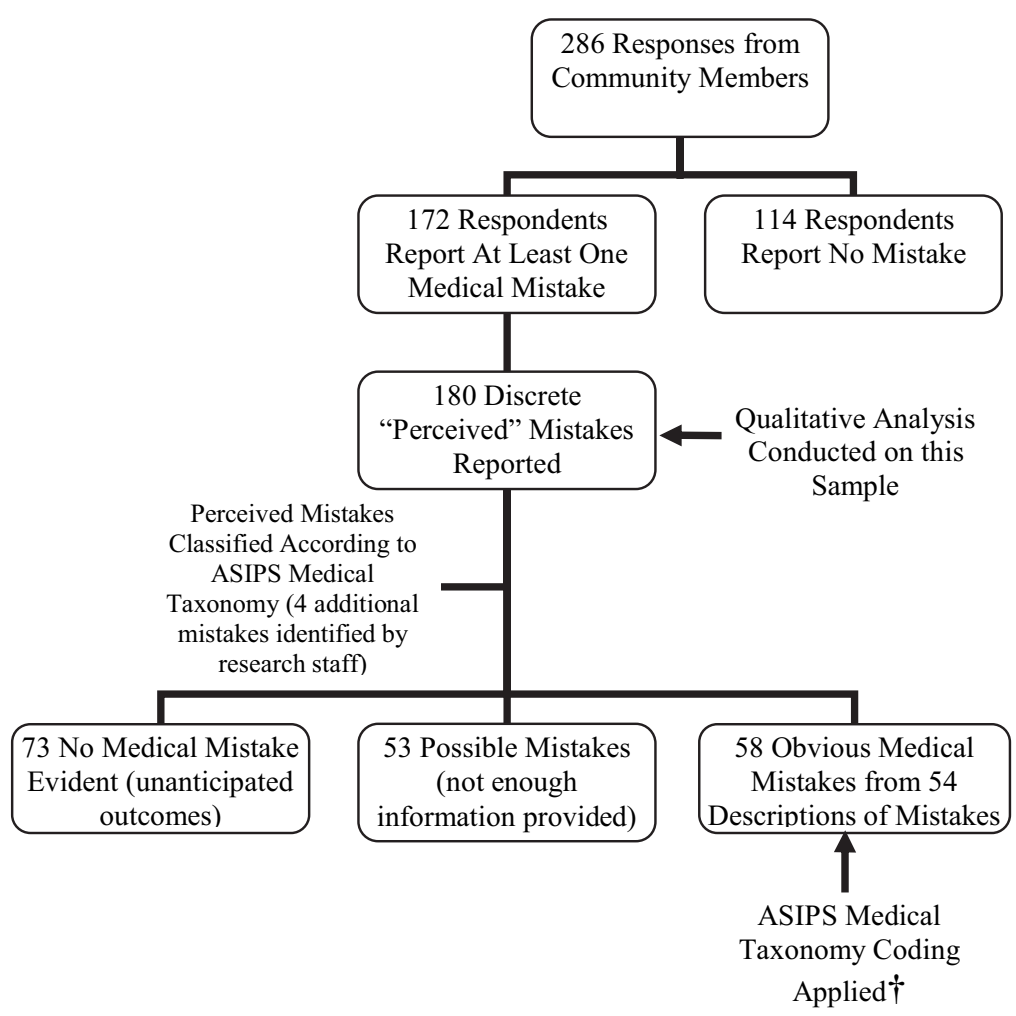

Figure 1. Response sample and analysis performed on each subset. †Applied Strategies for Improving Patient Safety $^{21}$.

Prescription drug ... was written for twice as often administration as wanted

Table 1. Characteristics of Reported Perceived Medical Mistakes $(\mathbf{n}=180)$

\begin{tabular}{lc}
\hline Characteristic & No. (\%) \\
\hline Gender of person involved & $106(59)$ \\
Female & $69(38)$ \\
Male & $5(3)$ \\
Missing & \\
Relationship to reporter of person involved & $88(49)$ \\
Self & $81(45)$ \\
Family member & $11(6)$ \\
Missing & \\
Age of person at time of event & $10(6)$ \\
Less than 18 years old & $33(18)$ \\
18 to 39 years old & $32(18)$ \\
40 to 54 years old & $52(29)$ \\
55 to 69 years old & $38(21)$ \\
70 years or older & $15(8)$ \\
Missing & \\
Reporters' perception of whether harm occurred & $155(86)$ \\
Yes & $19(11)$ \\
No & $6(3)$ \\
Missing &
\end{tabular}

[Nurse] was going to administer too much morphine ... daughter who is an $R N$ was with me, caught it

Postoperative complication ... ignored by Doctor leading to life-threatening situation

Many reports did not contain apparent medical mistakes but were descriptions of events with unanticipated outcomes:

A few days after my busband's fourth and final chemotherapy treatment, he contracted MRSA. This resulted in five months of hospitalization and three back surgeries. This infection was far more debilitating than the cancer of the bladder. ...

\section{Quantitative Findings}

The research staff coded the 54 reports that contained the obvious mistakes using the ASIPS DMO taxonomy. During this coding process, an additional 4 mistakes embedded within the reports were identified by the research team and coded (see Figure 1). Frequencies from the main taxonomy axes are presented in Table 3. Because this coding was applied to only the subset of reports, some of the numbers and percentages differ from the finding of the 180 reports that form the database for the qualitative analysis. 
Table 2. Description of Who Was Involved and Where Perceived Mistake Occurred $(\mathbf{n}=\mathbf{1 8 0}) *$

\begin{tabular}{lc}
\hline Characteristic & No. $(\%)$ \\
\hline Who was consulted about event & \\
Family member/friend & $137(76)$ \\
Doctor/clinician & $109(61)$ \\
Nurse & $46(26)$ \\
Hospital management & $40(22)$ \\
Patient representative & $29(16)$ \\
Pharmacist & $14(8)$ \\
Radiologist & $13(7)$ \\
No one & $12(7)$ \\
Other & $46(26)$ \\
Who was involved in the event & \\
Doctor/clinician & $144(80)$ \\
Nurse & $71(39)$ \\
Hospital staff & $59(33)$ \\
Specialist & $38(21)$ \\
Radiology & $27(15)$ \\
Laboratory & $22(12)$ \\
Office staff & $20(11)$ \\
Pharmacy & $11(6)$ \\
Other & $18(10)$ \\
Lhere the event occurred & \\
Local hospital & $79(44)$ \\
Local doctor's office & $64(36)$ \\
Urban/suburban doctor & $24(13)$ \\
Urban/suburban hospital & $26(14)$ \\
Other & $6(3)$ \\
\hline
\end{tabular}

* Percentages total more than $100 \%$ because questionnaire options were not mutually exclusive.

Mistakes occurred across a variety of settings, but were reported more frequently for hospitals $(n=19)$ and ambulatory offices $(n=12)$. In almost two thirds of reports, physicians were active participants involved in the mistake $(n=36)$ and both nonphysician providers and nursing staff were involved in 23 reports. Sixty-two percent $(n=36)$ involved a clinical event as the type of error (eg, surgery, lab test, diagnosis code), whereas communication errors and medication errors were each described in 23 reports.

Outcome interventions could not be determined in more than one third of all cases $(n=20) ; 14$ described medical mistakes that resulted in repeated procedures $(24.1 \%), 6$ in medication modifications $(10.3 \%)$, and 8 reports $(13.8 \%)$ did not require an intervention after the medical mistake.
Table 3. Research Staff Coding of 54 Patient Reports of Medical Errors Using the ASIPS DMO Taxonomy*

\begin{tabular}{|c|c|}
\hline Characteristic & No. (\%) \\
\hline \multicolumn{2}{|l|}{ Participants involved in mistake } \\
\hline Physicians & $36(62)$ \\
\hline Nonphysician providers and nurses & $23(40)$ \\
\hline Third party & $23(40)$ \\
\hline \multicolumn{2}{|l|}{ Mistake settings } \\
\hline Hospitals & $19(33)$ \\
\hline Ambulatory offices & $12(21)$ \\
\hline Emergency departments & $8(14)$ \\
\hline Outpatient diagnostic facilities & $6(10)$ \\
\hline Pharmacies & $6(10)$ \\
\hline Laboratories & $2(3)$ \\
\hline \multicolumn{2}{|l|}{ Type of mistake } \\
\hline Clinical event & $36(62)$ \\
\hline Communication errors & $23(40)$ \\
\hline Medication errors & $23(40)$ \\
\hline \multicolumn{2}{|l|}{ Cause of mistake } \\
\hline Clinical knowledge or skill & $7(12)$ \\
\hline Errors in judgment & $6(10)$ \\
\hline Distraction or inattention & $6(10)$ \\
\hline System malfunction, system not present & $3(5)$ \\
\hline Resource not available & $2(3)$ \\
\hline Failure in information retrieval & $1(2)$ \\
\hline Could not determine & $35(60)$ \\
\hline \multicolumn{2}{|l|}{ Outcome interventions } \\
\hline No intervention needed & $8(14)$ \\
\hline Repeated procedures & $14(24)$ \\
\hline Medication modification & $6(10)$ \\
\hline Could not determine & $20(35)$ \\
\hline \multicolumn{2}{|l|}{ Types of outcomes from mistake } \\
\hline No change in health status of patient & $24(41)$ \\
\hline Temporary impairment & $9(16)$ \\
\hline Permanent impairment & $4(7)$ \\
\hline Personal inconvenience & $9(16)$ \\
\hline Discomfort & $27(47)$ \\
\hline New diagnosis resulting from mistake & $4(7)$ \\
\hline Death & $4(7)$ \\
\hline Could not determine & $1(2)$ \\
\hline
\end{tabular}

* For any axis, more than 1 code can be assigned.

Actual cause of the mistake could not be determined in $60 \%$ of reports $(n=35)$; however, errors in clinical knowledge or skill seemed to be the cause of 7 mistakes, and errors in judgment and provider distraction or inattention each accounted for 6 mistakes.

In 24 cases (41\%), respondents did not experience a change in health status due to the mistake. However, 4 reports cited that a permanent impair- 
ment occurred and 27 respondents reported discomfort (46.5\%). Four deaths were reported.

\section{Qualitative Findings}

The qualitative analysis of all 180 reports yielded insight on the types of events that community members understood to be medical mistakes and to the type and severity of harm community members' believed they experienced as a result of the perceived mistake.

In addition to the obvious medical mistakes and those events with unanticipated outcomes, many reports included other related issues that were perceived to either be a medical mistake or to have resulted in harm.

\section{Violations of Trust}

Reports often included comments about lost faith or trust in integrity of medical professionals following the event:

... made me lose confidence in the Dr. and nurse

In several cases, patients discontinued treatment and/or relations with providers and then sought treatment elsewhere.

Although loss of trust was often the outcome of a mistake, several reports contained descriptions of a clinician's breach of confidentially or trust that led to a person feeling harmed by the incident:

Dr. was visiting [my family member]. [My family member] had a visitor, not immediate family, Doctor proceeded to tell visitor intimate details about [family member]

\section{Unmet or Unmatched Expectations Between Patients and Clinicians}

Several reports contained issues where expectations from the patient perspective were not met or were dismissed:

My father requested that he be tested for West Nile Virus. The doctor refused, saying that it was all publicity.

In other cases, the patient seemed to have knowledge or expectations that contradicted the provider's actions or judgments toward the medical problem:

[Injury] taking over 2 years to recover. Should have been over in 6 months, not 2 years.

\section{Lack of Satisfactory Communication Between Patient and Medical Professional}

Reports also indicated that patients believed the clinicians did not adequately communicate with them about their status or treatment plan, or that clinicians ignored documented histories, other information provided in medical records, or information provided verbally by patients.

Dr.'s nurse told me Dr. didn't need to see me. She said to just get some Claritin and I'd be $O K$.

\section{Manner or Deportment of Medical Professionals}

Reports noted the lack of personal attention to patients' needs, and lack of "caring" or unapologetic attitudes by clinicians:

I also was told by the doctor. . there was nothing wrong with me and I needed to calm down

\section{Types of Harm Reported}

Community members' reported physical pain including physical impairment, difficulty with movement, and physical discomfort:

He bas constant back pain.... The quality of bis life has been severely affected

Also described in the reports was emotional or mental harm including stress and frustration. Emotional harm was either specifically mentioned or implied by using other words or phrases including stress, intense suffering, anguish, and agony:

\section{... caused her great worry and anxiety}

In addition, reports described financial harm either directly or implied through descriptions of loss of income, lost time from work, and additional tests and trips to the doctor's office:

It forever changed their lives and has taken all savings and retirement

Many reports included multiple types of harm. Emotional harm was often combined with either financial or physical harm:

Emotionally and financially due to added tests and trips....

He has a scar that is 4 inches long. I also think there are emotional scars

The severity of reported harm ranged from extreme harm (ie, death or loss of function) to minimal harm:

He no longer has sight in bis left eye

She died, that's about as harmed as you can get

Only took longer to get well

\section{Discussion}

This study combines CBPR with mixed methods in a PBRN to collect and describe rural community members' experiences and descriptions of harm 
from medical mistakes. The mixed methods including quantitative and qualitative analyses provided a rich description of the medical mistake reports we received. The quantitative analysis using a welldescribed patient-safety taxonomy was designed to allow for an in-depth report on patient descriptions of medical mistakes and the potential for comparison to reports from physicians and office staff. However, it seems that without more clear direction of what to report, community members may not provide enough standard information to conduct cause analysis or report outcomes. The qualitative analysis of the "stories" we received allowed us to gain a broad understanding of what community members' understand to be medical mistakes. ${ }^{23,26}$

As in previous studies, community members reported a broad range of issues as medical mistakes including unanticipated outcomes, communication difficulties and violations of trust. ${ }^{6,27-29}$ At least $41 \%$ of the 180 reported mistakes that we received were not judged to be medical mistakes when coded with a taxonomy designed to specifically describe medical errors. Many reports included related issues that seemed to impact the patient-clinician relationship (eg, violations of trust between the patient and the clinician, lack of communication, and perceived uncaring or inattention by clinicians and their medical staff). Such issues can have profound implications for clinical care particularly when there are unanticipated outcomes.

Community members' ideas about what constitutes a medical mistake seem to be quite different from the medical world as evidenced by our finding that one third of the reports included only an unanticipated outcome and included no evidence of a mistake. Community members may lack the sophisticated medical knowledge necessary to define and describe a medical mistake, particularly related to cause. We have found that it required a significant amount of education and practice from our research team to reliably read error reports and code them consistently. The differences could reflect a communities' broader view of medical mistakes, requiring the medical community to consider issues previously relegated to the cause of unanticipated results (or bad luck) as important factors in patient safety research. Certainly, patient perceptions around these issues are important in current and ongoing patient safety research.
The content of our findings are not drastically different from what others have reported on harm from medical mistakes. As described previously in the literature, survey respondents in this study described harm from medical mistakes as physical pain, emotional pain, and financial loss. ${ }^{10}$ However, we found that the reports we received had more depth of emotion than found in standard reporting mechanisms and we found that the emotional and financial impact from perceived medical mistakes was a major outcome reported by our respondents. Our use of anonymous, open-ended questions in this study, may have given patients who wanted to talk about these issues, both permission and a venue.

Although we were not surprised that respondents indicated a lack of trust in their clinician as an outcome of a mistake, ${ }^{9,16}$ we were intrigued by the reports that described respondents feeling harmed by clinicians who violated their trust, by clinicians who did not respond appropriately to their needs, and by the reports that noted unsatisfactory communication as the medical mistake. ${ }^{30}$ Patients may be better able to describe a disruption in the patient-physician relationship than they are to define an actual medical mistake.

This study identifies some very practical implications for clinicians. The fact that community members believed that a medical mistake had occurred may be the result of insufficient communication by the provider or health care system. We frequently saw that the perception of how the patient was treated by the medical community after an unexpected outcome affected the perception of the event. Several reports indicated that if a medical professional took action to "right a wrong," the respondent seemed to be more forgiving. Thus, when peoples' concerns are not addressed or validated, they may be more likely to perceive an error has occurred. ${ }^{10}$

Establishing an open and honest communication system around medical mistakes is a difficult process. Although $80 \%$ of the reports indicated that a clinician or doctor was involved, only $61 \%$ talked to the clinician or doctor. Fostering an environment for patients to talk openly with clinicians about medical mistakes and giving clinicians the opportunity to review each case with the patient or family and offer education on the natural course of disease, complications, and unpreventable adverse events, may alter the patient's perception of "mis- 
take." ${ }^{3,31-33}$ Physicians and office staff need to be vigilant in communication in cases of medical mistakes and unanticipated outcomes. An open reporting system for patient concerns or complaints complete with a safe feedback mechanism may be an important component of clinical care in rural communities.

This study demonstrates that participatory research methods are effective in geographically separate rural communities. Working collaboratively with the research team, CAC members shaped the survey language to be understandable by the community. Through an iterative process, we were able to engage the CAC members in the analysis and interpretation of the data. The HPRN CAC offered insight into a distribution method that would reach the greatest number of community residents, and as a result, we were able to obtain 180 descriptions of medical mistakes allowing us to understand the type of mistakes and harm experienced as well as to describe the impact of perceived medical mistakes on the clinician-patient relationship.

In many cases, the reports we received were rich with information and many conveyed strong emotional reactions. For example, one respondent wrote: "The suffering my mother endured was unconscionable." We believe that community members within the HPRN region felt comfortable entrusting us with their stories. Although open-ended questions in some cases resulted in incomplete data for the taxonomy coding, it seems that anonymous survey distribution via the media was a viable and cost-effective way to collect qualitative medical mistake data from community members.

Our study has several limitations. In one third of the reports, we were not given enough information to determine whether a true mistake had occurred or to adequately understand the nature of the mistake. Thus, we were unable to code these cases using the patient safety taxonomy. Without more description of cause, our ability to inform interventions becomes greatly reduced. However, this survey was not designed to determine cause. Future patient reporting systems may want to ask for specific information describing the cause of the event or collect contact information to allow for follow-up data collection. Second, although using an anonymous survey design distributed in local newspapers gave us rich information on the quality of the error experience of our participants, our distribution method does not allow us to make any comments about the frequency of medical mistakes in these communities.

Conducting CBPR across a large geographical area can be at times logistically challenging. At multiple times during this project (eg, distributing the data, joint coding sessions, discussion of results), it was necessary for us to hold face-to-face meetings (5) between the research group and the CAC and countless phone calls to individual CAC members. Furthermore, the CAC members were not paid for their work on this study and most of the members have full-time jobs. Thus, scheduling multiple people who live and work hundreds of miles apart was at times difficult. However, we believe that the additional effort necessary to conduct this study using a CBPR approach was greatly exceeded by the CAC's contributions. The CAC members validated previous research and helped to ground this project and our findings in real patient experience. The CAC is currently discussing methods for disseminating these findings back into the community in an honest, respectful, and helpful manner.

We acknowledge the following members of the HPRN CAC for their contributions to the methodology, analysis, and interpretation of findings: Shirley Cowart, Michael Hernandez, Connie Haynes, Garry Haynes, Ned Norman, Mary Rodriquez, Cameron Walsh, Kathy Winkelman, and Steve Winkelman.

\section{References}

1. Advances in Patient Safety: From Research to Implementation. Vols. 1-4, AHRQ Publication Nos. 050021 (1-4). Agency for Healthcare Research and Quality, Rockville, MD; February 2005.

2. Layde PM, Cortes LM, Teret SP, et al. Patient safety efforts should focus on medical injuries. JAMA 2002;287:1993-7.

3. Vincent C, Coulter A. Patient safety: what about the patient? Qual Saf Health Care 2002;11:76-80.

4. Phillips R, Dovey S, Graham D, Elder N, Hickner J. Learning from different lenses: reports of medical errors in primary care by clinicians, staff and patients. J Patient Safety 2006:2(3):140-146.

5. Schoen C, Osborn R, Huynh PT, et al. Taking the pulse of health care systems: experiences of patients with health problems in six countries. Health Aff (Millwood) 2005;W5:509-25.

6. Kuzel AJ, Woolf SH, Gilchrist VJ, et al. Patient reports of preventable problems and harms in primary health care. Ann Fam Med 2004;2:333-40.

7. Elder NC, Jacobson C, Zink T, Hasse L. How experiencing preventable medical problems changed 
patients' interactions with primary health care. Ann Fam Med 2005;3:537-44.

8. Duclos CW, Eichler M, Taylor L, et al. Patient perspectives of patient-provider communication after adverse events. Int J Qual Health Care 2005; 17 : 479-86.

9. Safran DG, Taira DA, Rogers WH, Kosinski M, Ware JE, Tarlov AR. Linking primary care performance to outcomes of care. Soc Sci Med 1995;41: $1639-45$.

10. Harris LE, Luft FC, Rudy DW, Tierney WM. Correlates of health care satisfaction in inner-city patients with hypertension and chronic renal insufficiency. J R Soc Health 1992;112:64-7.

11. Sullivan $M$. The new subjective medicine: taking the patient's point of view on health care and health. Int J Qual Health Care 2003;15:309-18.

12. Blendon R, DesRoches C, Brodie M, et al. Views of practicing physicians and the public on medical errors. N Engl J Med 2002;347:1933-40.

13. Gandhi T, Weingart S, Borus J, et al. Adverse drug events in ambulatory care. N Engl J Med 2003;348: $1556-64$.

14. Keating N, Green D, Kao A, Gazmararian J, Wu V, Cleary P. How are patients' specific ambulatory care experiences related to trust, satisfaction, and considering changing physicians? J Gen Intern Med 2002; 17:29-39.

15. Keating N, Gandhi T, Orav J, Bates D, Ayanian J. Patient characteristics and experiences associated with trust in specialist physicians. Arch Intern Med 2004;164:1015-20.

16. Macaulay AC, Commanda LE, Freeman WL, et al. Participatory research maximizes community and lay involvement. BMJ 1999;319:774-8.

17. Green LW, George MA, Frankish CJ, et al. Study of participatory research in health promotion in Canada. Ottawa, Canada: Royal Society of Canada, 1995.

18. Macaulay A, Nutting P. Moving the frontiers forward: incorporating community-based participatory research into practice-based research networks. Ann Fam Med 2006;4:4-7.

19. Westfall J, VanVorst R, Main D, Herbert C. Community-based participatory research in practicebased research networks. Ann Fam Med 2006;4:814.

20. Addison R. A grounded hermeneutic editing approach. In: Crabtree BF, Miller WL, editors. Doing qualitative research. 2nd ed. Thousand Oaks, CA: Sage Publications; 1999.

21. Fernald D, Pace W, Harris D, West D, Main D, Westfall J. Event reporting to a primary care patient safety reporting system: a report from the ASIPS collaborative. Ann Fam Med 2004;2:327-32.

22. Westfall JM, Fernald DH, Staton EW, VanVorst R, West D, Pace WD. Applied strategies for improving patient safety: a comprehensive process to improve care in rural and frontier communities. J Rural Health 2004;20:355-62.

23. The ASIPS Collaborative. Dimensions of medical outcome. The ASIPS-Victoroff taxonomy 2003. Available at www.errorsinmedicine.net/taxonomy.

24. Pace W, Fernald D, Harris D, Dickinson L, et al. Developing a Taxonomy for Coding Ambulatory Medical Errors: A Report from the ASIPS Collaborative. In: Henrikson $\mathrm{K}$, Battles J, Marks E, et al, editors. Advances in Patient Safety: From Research to Implementation. Vol. 2, Concepts and methodology. Rockville, MD: Agency for Healthcare Research and Quality; 2005.

25. Harris D, Westfall J, Fernald D, et al. Mixed Methods Analysis of Medical Error Event Reports: A Report from the ASIPS Collaborative. In: Henrikson $\mathrm{K}$, Battles J, Marks E, et al, editors. Advances in Patient Safety: From Research to Implementation. Vol. 2, Concepts and methodology. Rockville, MD: Agency for Healthcare Research and Quality; 2005.

26. Newman TB. The power of stories over statistics. BMJ 2003;327:1424-7.

27. Weingart S, Pagovich O, Sands D, et al. What can hospitalized patients tell us about adverse events? Learning from patient-reported incidents. J Gen Intern Med 2005;20:830-6.

28. Gallagher T, Waterman A, Ebers A, Fraser V, Levinson W. Patients' and physicians' attitudes regarding the disclosure of medical errors. JAMA 2003;289:1001-7.

29. Woods D, Cook R. Mistaking Error. In: Youngberg B, Hatlie M, editors. The Patient Safety Handbook. Sudbury, MA: Jones and Bartlett; 2003.

30. Bernstein M, Potvin D, Martin DK. A qualitative study of attitudes toward error in patients facing brain tumour surgery. Can J Neurol Sci 2004;31: 208-12.

31. Mazor KM, Simon SR, Gurwitz JH. Communicating with patients about medical errors: a review of the literature. Arch Intern Med 2004;164:1690-7.

32. Hickson GB, Clayton EW, Githens PB, Sloan FA. Factors that prompted families to file medical malpractice claims following perinatal injuries. JAMA 1992;267:1359-63.

33. Hickson GB, Federspiel CF, Pichert JW, Miller CS, Gauld-Jaeger J, Bost P. Patient complaints and malpractice risk. JAMA 2002;287:2951-7. 